\title{
Pain Management in Interventional Radiology: An Introduction
}

\author{
Jason R. Bauer and Charles E. Ray, Jr.
}

\section{THE PROBLEM OF PAIN}

The management of pain continues to be one of the most vexing of problems for health practitioners worldwide. The pain response is difficult to manage for multiple reasons: responses vary widely in their mechanism of action as well as anatomic location; individual responses to pain are variable; secondary gains can be had by individuals falsely claiming to be in pain; cultural differences in description of pain and the willingness to admit to a pain response exist; even responses by the health care provider to a patient in pain varies widely. For these reasons, each and every patient who presents with pain, or in whom we as health care providers are likely to produce a painful response during a procedure, has a widely variable and largely unpredictable pain response.

Traditional medicine (so-called Western medicine) has demonstrated great advances in the management of pain; however, the advances have been relatively few and far between. Opium was cultivated for recreational use by the Sumerians nearly 5,000 years before it was first introduced specifically for medicinal purposes (1). It was not until 1680 that opium was introduced in England as Laudanum, a combination of opium, herbs, and sherry, specifically to be used for medicinal purposes. Although likely poorly understood at the time, the pain-relieving effects of opium must have significantly contributed to the use of opium as a medicinal agent. It is amazing, actually, that a plant discovered nearly five centuries ago remains the mainstay for pain relief throughout nearly all cultures and medical systems worldwide.

The next great advance in pain control was the discovery of agents used for general anesthesia during operations. The first public display of the effect of general anesthesia was performed at the Massachusetts General Hospital in 1846 (2). Dr. John Warren, a preeminent surgeon of the day, removed a vascular tumor from the jaw of a patient. This surgery was performed in conjunction with William Morton, a local dentist, serving as an anesthetist, by using an ether-soaked sponge under a glass dome. Upon awaking at the end of the procedure, the patient claimed he had no pain during the operation, to 
Cambridge University Press

978-0-521-86592-o - Pain Management in Interventional Radiology

Charles E. Ray

Excerpt

More information

which Dr. Warren proclaimed to the audience (legend has it), "Gentlemen, this (anesthesia) is no humbug!"

One must take note that these two greatest advancements in the history of pain control - narcotics and general anesthesia - occurred a remarkably long time ago. Clearly, advancements have been made since then with regards to pain control, and the adjustments to the use of both narcotics and general anesthesia have allowed significant advancements in pain management. Other advancements, such as postoperative pain control with patient-controlled analgesia [described by Sechzer in 1971(3)] and the administration of intraprocedural sedation and analgesia, are the more recent improvements that have been major factors in pain management. However, if one regards the changes in other fields of medicine that have occurred since 1846 (antibiotics, chemotherapy for cancer, open heart surgery, transplant surgery, etc.), one is struck by the relative paucity of new methods of pain control, particularly long-lasting or "curative" pain control, that have occurred over the past several decades.

Western medicine has had a difficult time throughout its history in dealing with a patient in pain. One needs look no further than the number and types of alternative therapies available to individuals to treat pain. Chiropractic, napropathic, and homeopathic medicine forms largely deal with patients in pain, as does acupuncture, massage therapy, craniosacral therapy, and many others. Are the successes of these alternative medicine systems in treating patients in pain in part due to our failure as traditional medicine providers?

If Western medicine has largely fallen short when dealing with the patient in pain, interventional radiology (IR) has in many ways ignored the problem altogether. Except for giving a touch of sedation and analgesia during our procedures, or occasionally treating the patient in pain with a nerve block, spinal procedure, or embolization, IR provides very little attention to the patient in pain. This is perhaps historical as much as anything; because IR started as a diagnostic modality where our contact with any given patient extended for only an hour or two; the need to understand the pain response and the best way to treat it was underappreciated. It has only been because we as interventionalists have become more involved with patients as care providers rather than proceduralists that the need to understand patients in pain has become more vital to our daily practice.

\section{PAIN IN MEDICINE AND SOCIETY}

Pain, and the treatment of pain, is an enormous medical/social issue. From a societal standpoint, the cost of pain is tremendous. One study evaluated nearly 30,000 workers in the U.S. workforce, and estimated that the total cost of lost productivity in the workplace because of common pain conditions (headache, arthritis, back pain, and other musculoskeletal pain) was $\$ 61.2$ billion per year (4). The same study noted that the majority of the lost productivity was due to reduced performance while at work, rather than taking time off.

The costs (in dollars) associated with pain control are nearly impossible to accurately calculate. One study evaluated 373 cancer patients, nearly $40 \%$ of who reported having some sort of cancer-related pain (5). Of these patients, three-quarters incurred a pain-related expense, averaging $\$ 891$ per month 
Cambridge University Press

978-0-521-86592-o - Pain Management in Interventional Radiology

Charles E. Ray

Excerpt

More information

\begin{tabular}{|ll|}
\hline Table 1. Results from PubMed Search, January 2007 \\
Search Term & Number of Articles Returned \\
\hline "Pain" & 341,298 \\
"Pain," limited to last one year & 18,569 \\
"Pain, interventional radiology," limited to last one year & 19 \\
\hline
\end{tabular}

related specifically to pain control. Other studies have evaluated common conditions such as low-back pain, which in terms of both direct costs and indirect costs (lost productivity, etc.), is likely the most costly medical problem facing the medical establishment in the United States. In 1991, low-back pain was estimated to cost the United States $\$ 25$ billion in direct costs alone $(6,7)$. It has also been estimated that $80 \%$ of all adults at some point in their lifetime will seek medical care of some sort for low-back pain and that a third of all disability costs in the United States are due to the same problem $(6,8)$. Clearly, pain management represents a huge percentage of both the time and dollars spent on health care in this country.

Medically, the search for improvement in pain management is also consuming when one considers the amount of research time (and dollars) spent on pain control. The importance of research into pain and the management of pain are revealed when one considers the attention given to pain by the National Institutes of Health (NIH). The NIH has previously focused attention on pain research with the development of multiple committees, such as the NIH Pain Research Consortium, the NIH Extramural Pain Staff Workgroup, and the NIH Pain Interest Group, specifically charged with attempting to drive more research into pain control (9).

Also illustrative of the research efforts regarding pain management, over twenty journals are dedicated to pain, with such widely varied titles as "Pain," "Journal of Orofacial Pain," and "Molecular Pain" (whatever THAT may be!). At the time of the manuscript preparation, a PubMed search was performed with the single keyword "pain" (Table 1). A total of more than 340,000 articles were returned. When limited to manuscripts published within the past year, nearly 19,000 articles were returned. Amazingly, when the keywords "interventional radiology" were added to the search, only 19 articles were returned for a one-year time period. Clearly, we as interventionalists have some catching up to do with regard to understanding the importance of pain management for our patients.

\section{IR AND PAIN MANAGEMENT}

The role of IR in pain management varies widely from institution to institution, from practice to practice, and from operator to operator. To try to define pain management as a major or minor player in the field as a whole is impossible, and each individual practitioner must determine the needs of the patient, the referral patterns for their individual practice, and their own desire to become involved with pain management to decide what amount of time and effort will 
Cambridge University Press

978-0-521-86592-o - Pain Management in Interventional Radiology

Charles E. Ray

Excerpt

More information

be spent on the management of pain. At one of the authors' (CER) institutions, for instance, the number of pain procedures we have performed over the past two years has increased by a factor of 2.7 , largely due to increased involvement with low-back pain management. Parenthetically, the number of procedures performed for an indication of pain still only accounts for $5 \%$ of all the cases performed at that one institution.

One instance in which we all must be involved in pain management, however, is in controlling the pain (and anxiety) that we specifically cause during procedures performed for other reasons. It is in this management of intraprocedural pain that we all have a common interest. Intraprocedural pain control is important for several reasons. As described by the American Society of Anesthesiologists in their practice guidelines for sedation and analgesia by nonanesthesiologists (10), the goals of intraprocedural sedation and analgesia are twofold. First, it "... allows patients to tolerate unpleasant procedures by relieving anxiety, discomfort, or pain." Second, “. . in children and uncooperative adults, sedation-analgesia may expedite the conduct of procedures that are not particularly uncomfortable but that require that the patient not move" (10). Control of intraprocedural pain is important for other reasons as well, particularly if the same patient is going to return to the same interventionalist for repeat procedures. Nothing will dissuade an individual from returning for repeat procedures quite like a previously painful experience!

Pain management in IR has evolved over time. Intially, nearly completely ignored except for the use of local anesthestics at the skin entry site, increasing attention was paid to pain control needed during the procedure itself, with widespread use of intraprocedural sedation and analgesia. Procedures specifically designed to treat a patient's underlying pain, such as embolization or ablation of painful osteolyses, were developed. Finally, again with our increasing involvement in patient care, further attention has been given to postprocedural management of pain. For these reasons, many IR divisions are now considered along with anesthesia, neurology, neurosurgery, oncology, and orthopedic medicine, as one of the "pain services."

The use of intra-arterial injection of local anesthesthetics provides an example of the evolution of pain management in IR - a brief synopsis is given in the following paragraphs.

\section{USE OF INTRA-ARTERIAL LOCAL ANESTHETICS}

The use of intra-arterial anesthetics in the management of pain is relegated to few uses today. From a historical perspective, the use of these agents intravascularly was linked to the evolution of angiography and the pain resulting from contrast injections $(11,12)$. There has been a reemergence of intra-arterial analgesia in IR largely as a result of increased interest in transcatheter tumor therapy.

As early as 1939, adjuvant injection of medications to limit pain experienced during peripheral angiography was explored. Procaine hydrochloride (Novocaine) injection was mentioned by Dimitza and Jaegar with apparently improved pain during peripheral angiography (11). In 1982, Cranston performed a double-blinded placebo-controlled trial of thirty-four patients having 
Cambridge University Press

978-0-521-86592-o - Pain Management in Interventional Radiology

Charles E. Ray

Excerpt

More information

peripheral angiography (13). Subjective and objective evaluations were performed using verbalization and movement. One milliliter of $2 \%$ lidocaine was mixed for each $10 \mathrm{ml}$ of Conray-60 used. Seventeen patients received lidocaine during the first injection of contrast, thirteen during the second injection, and four received two injections without contrast. All patients who received lidocaine in the first injection reported subjective improvement, and there were no associated complications.

The debate regarding effectiveness or need for intra-arterial lidocaine administration during peripheral angiography is now all but moot. With the evolution of digital subtraction angiography (using less contrast at lower rates) and low osmolality contrast agents such as Visipaque (GE Healthcare, Cork, Ireland), few patients experience symptoms so intolerable as to warrant intraarterial lidocaine.

When considering transcatheter tumor therapy for malignant tumors, such as hepatocellular carcinoma in the liver, or benign tumor management, such as symptomatic leiomyomata in the uterus, pain management strategies have included intra-arterial lidocaine injection. In 1990, Molgaard et al. injected intra-arterial lidocaine in the hepatic artery prior to and during Transcatheter arterial Chemoembolization (TACE) in 45 patients (14). Analgesic requirements during and following the procedure were compared with that in 20 patients previously treated without intra-arterial lidocaine (14). They found a remarkable decrease in the amount of medication required during (98.9\% decrease in narcotic dose) and after (75\% fewer individuals requiring a patient-controlled analgesia pump) the procedure in those who received lidocaine. Lee et al. evaluated the importance of timing of intra-arterial lidocaine injection (15). In 113 consecutive patients, three groups of patients (no lidocaine, lidocaine just prior to TACE, and lidocaine following TACE) were evaluated by quantifying the mean dose of analgesic and subjective pain score. There was a statistically significant difference in both the amount of analgesics used and the pain score; those patients who received $100 \mathrm{mg}$ of lidocaine prior to TACE used fewer narcotics and reported a lower pain score than those receiving similar treatment administered after delivery of the chemotherapeutic agent. The authors reported few complications, with only one patient experiencing transient hypotension.

Intra-arterial lidocaine has also recently been investigated during the endovascular treatment of uterine fibroids. Embolization in this setting notoriously results in a postembolization syndrome, punctuated by pain and cramping. Postprocedural pain in this setting may extend the length of hospital stay and lead to return visits. Similar to TACE, pain is thought to result from leiomyoma ischemia, spasm, and parenchymal swelling (16). However, uterine arteries do not appear to respond to lidocaine in the same way as hepatic arteries following injection of intra-arterial lidocaine; this may be due to their need to meet the demands of a gravid uterus (17). Keyoung et al. injected $200 \mathrm{mg}$ of lidocaine in 10 consecutive patients (while eight received placebo) prior to uterine artery embolization (UAE) for leiomyomata (17). Lidocaine was found to significantly improve subjective pain reported by patients but not the amount of analgesia required following the procedure. More importantly, moderate to severe vasospasm was noted in seven of ten patients after lidocaine; none of the placebo patients demonstrated such spasm, resulting in early termination of the study. Vasospasm due to lidocaine injection may therefore 
Cambridge University Press

978-0-521-86592-o - Pain Management in Interventional Radiology

Charles E. Ray

Excerpt

More information

contribute to a higher treatment failure rate and therefore is not commonly used during UAE.

Intra-arterial lidocaine was once common during peripheral angiography; its safety profile has been supported during years of use for this application. With overall few reported complications, intra-arterial lidocaine injection prior to tumor embolization provides practitioners with a safe strategy to achieve better patient comfort during and after embolization procedures.

\section{CONCLUSIONS}

"Pain management continues to be the most difficult problem facing medicine today." This statement is debatable, but arguments against it are largely due to a matter of degree and opinion, not of underlying substance. The patient in pain is ubiquitous, regardless of culture, geographic location, socioeconomic status, sex, race, or any other variable of which we can think.

IR can play a major role in pain management and is unique as a field in that we can be responsible for controlling patients' pain as a primary goal of therapy, or in controlling the pain that we cause during our procedures. This book is organized in such a way, with chapters on intraprocedural and postprocedural pain management, and multiple chapters on procedures performed specifically to treat underlying processes that may be responsible for the pain response. Special attention is given to spinal procedures, although other publications provide a more in-depth overview of spinal procedures. The goal of this book is to provide an overview of pain management in IR and introduce concepts that can be used on a daily basis in the interventional suite to better provide pain management for our diverse group of patients.

\section{REFERENCES}

1. opiods.com/timeline - accessed January 2007.

2. http://neurosurgery.mgh.harvard.edu/History/gift.htm - accessed January 2007.

3. Sechzer PH. Studies in pain with the analgesic-demand system. Anesth Analg, 1971;50: 1-10.

4. Stewart WF, Ricci JA, Chee E, Morganstein D, Lipton R. Lost productive time and cost due to common pain conditions in the US workforce. JAMA, 2003;290: 2443-54.

5. Fortner BV, Demarco G, Irving G, et al. Description and predictors of direct and indirect costs of pain reported by cancer patients. J Pain Symptom Manage, 2003;25: 9-18.

6. Bratton RL. Assessment and management of acute low back pain. Am Fam Physician, 1999;60: 2299-308.

7. Frymoyer JW, Cats-Baril WL. An overview of the incidences and costs of low back pain. Orthop Clin North Am, 1991;22: 263-71.

8. Kuritzky, Carpenter D. The primary care approach to low back pain. Prim Care Rep, 1995;1: 29-38.

9. http://www.ninds.nih.gov/find_people/nands/council_minutes_may1998.htm - accessed January 2007.

10. Practice guidelines for sedation and analgesia by non-anesthesiologists. An updated report by the American Society of Anesthesiologists Task Force on sedation and analgesia by non-anesthesiologists. Anesthesiology, 2002;96: 1004-17. 


\author{
Cambridge University Press \\ 978-0-521-86592-0 - Pain Management in Interventional Radiology \\ Charles E. Ray \\ Excerpt \\ More information
}

11. Lindbom A. Arteriography of the lower limb in the living subject. Acta Radiol [suppl], 1950;80: 9-15.

12. Bjork L, Erikson U, Ingelman B. Clinical experiences with a new type of contrast medium in peripheral angiography. Am J Roentgenol, 1969;106: 418-24.

13. Cranston PE. Lidocaine analgesia in peripheral angiography: a confirmation of effectiveness. South Med J, 1982;75: 1229-31.

14. Molgaard CP, Teitelbaum GP, Pentecost M, et al. Intra-arterial administration of lidocaine for analgesia in hepatic chemoembolization. JVIR, 1990;1: 81-5.

15. Lee SH, Hahn ST, Park SH. Intra-arterial lidocaine administration for relief of pain resulting from trans arterial chemoembolization of hepatocellular carcinoma: its effectiveness and optimal timing of administration. Cardiovasc Invervent Radiol, 2001;24(6): 368-71.

16. Cibils LA. Response of human uterine arteries to local anesthetics. Am J Obstet Gynecol, 1976;126: 202-10.

17. Keyoung JA, Levy EB, Roth AR, et al. Intra-arterial lidocaine for pain control after uterine artery embolization for leiomyomata. JVIR, 2001;12: 1065-9. 


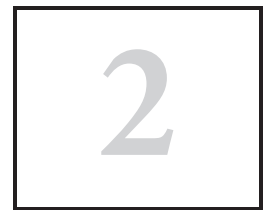

\title{
Clinical Evaluation of Low-Back Pain
}

\author{
Anthony P. Dwyer, Curt Freudenberger, and Vikas V. Patel
}

\section{INTRODUCTION}

The goal of this chapter is to explain the mindset and thought processes of the clinician evaluating a patient presenting with symptoms of low-back and leg pain.

The accurate clinical evaluation of the symptom of low-back pain is essential to the successful management of patients presenting with this problem, and interventional radiology (IR) plays an important role in the process.

It is vital to recognize that low-back pain is only a symptom and not a diagnosis or a disease entity in and of itself. Unfortunately, the successful management of patients with low-back pain is difficult because of the diversity of the pathologies producing low-back pain. There are many pathologies that can produce the symptom of low-back pain, and its treatment is very difficult or impossible unless one can locate and understand the causative pathology. The key to successful management of patients presenting with low-back pain is to identify and understand the pathological cause of their presenting symptoms of low-back pain, and to direct therapy toward the underlying cause rather than solely relieve the symptom of pain.

Although low-back pain typically arises from spinal causes, it must be remembered that there are pathologies outside of the spinal column that can present with low-back pain. These etiologies are typically considered only after a spinal column abnormality has been excluded.

A useful classification of the causes of low-back pain is the classification scheme described by Macnab et al. (1). These are presented in Table 1.

Another diagnostic hurdle is the difficulty in determining whether the pathologies identified on spinal imaging are the cause of the low-back pain or coincidental to it, as a significant percentage of asymptomatic patients have pathology identified on spinal imaging [especially on magnetic resonance imaging (MRI)] (2). Not infrequently, clinical examinations, patient symptoms, and imaging studies are discordant. Spinal interventions are often requested as a method by which to determine whether or not the pathology 
Cambridge University Press

978-0-521-86592-o - Pain Management in Interventional Radiology

Charles E. Ray

Excerpt

More information

\begin{tabular}{|l|}
\hline Table 1. Classification Scheme for the Causes of Low-back Pain \\
\hline Viscerogenic \\
Vascular \\
Neurogenic \\
Psychogenic \\
Spondylogenic \\
Traumatic \\
Infectious \\
Neoplastic \\
Metabolic \\
\hline Source: Macnab et al. (1).
\end{tabular}

noted on the imaging study is responsible for the patients' pain. This subject is beyond the scope of this chapter and is discussed in the individual, procedurally oriented chapters that follow.

This chapter will concentrate on the clinical evaluation of the more common spinal pathologies that affect the vast majority of low-back pain patients in clinical practice. Oftentimes, regardless of the underlying cause (e.g., traumatic, infectious), the symptom of pain itself is caused only after significant degenerative changes have occurred within the spinal column.

\section{LUMBAR PAIN OVERVIEW}

By definition, somatic pain occurs with noxious stimuli to musculoskeletal structures, visceral pain occurs with noxious stimuli to an organ, and neurogenic pain occurs with noxious stimuli to axons and cell bodies. Referred pain is felt at a location away from the site of the causative pathology, with the site of pain being innervated by nerves different than the structure causing the referred pain. It must be remembered that virtually any structure receiving innervation is a potential source of pain when it is at the site of pain-producing pathology.

With lumbar pain, the causative pathology usually involves the structures of the disc and facet joints involved in degenerative cascade, as described in depth by Yong-Hing and Kirkaldy-Willis (3). Sources of lumbar pain are outlined in Table 2 (4).

Lumbar pain can be axial (central lumbar) or may radiate from the spine. The latter cause is felt as deep, aching, poorly localized pain usually in the buttocks and thighs. This latter constellation of symptoms is referred to as "somatic referred pain." Radicular pain arises from involvement of the spinal nerves with inflammation, irritation, and compression, producing a sharper pain localized in the radicular distribution of the involved nerve root. Radicular pain is often associated with objective neurological deficits, the most notable of which are sensory change and muscle weakness. Clinical studies have 
Cambridge University Press

978-0-521-86592-0 - Pain Management in Interventional Radiology

Charles E. Ray

Excerpt

More information

\section{Table 2. Anatomic Sources of Lumbar Pain}

\section{Vertebra}

Disc

Facet joints

Sacroiliac joints

Muscles

Ligaments

Dura

Source: Van Akkerveeken et al. (4).

established that nerve root compression alone does not produce radicular pain and that the nerve root must first be inflamed in order for compression to produce pain. These studies also confirmed the outer annulus as the common site of lumbar pain, with the facet joint capsule only occasionally being a cause (Figure 1) (5).

The vast majority of low-back pain is mechanical in nature and is usually related to spinal degeneration, or subclinical episodes of "wear and tear" that are aggravated intermittently by episodes of trauma. This "degenerative cascade," as described by Yong-Hing and Kirkaldy-Willis (3), is ubiquitous; its extent and severity is multifactorial, such as genetic predisposition, smoking, and occupational loads to the lumbar spine. The degenerative cascade produces degenerative joint changes in the articular cartilage of the facet joints, loss of hydration of the intervertebral disks with concomitant loss of stability and resistance to torsion, eventually leading to radial

Figure 1. Possible sites of lower back pain. (From Kuslich and Ulstrom (5)).

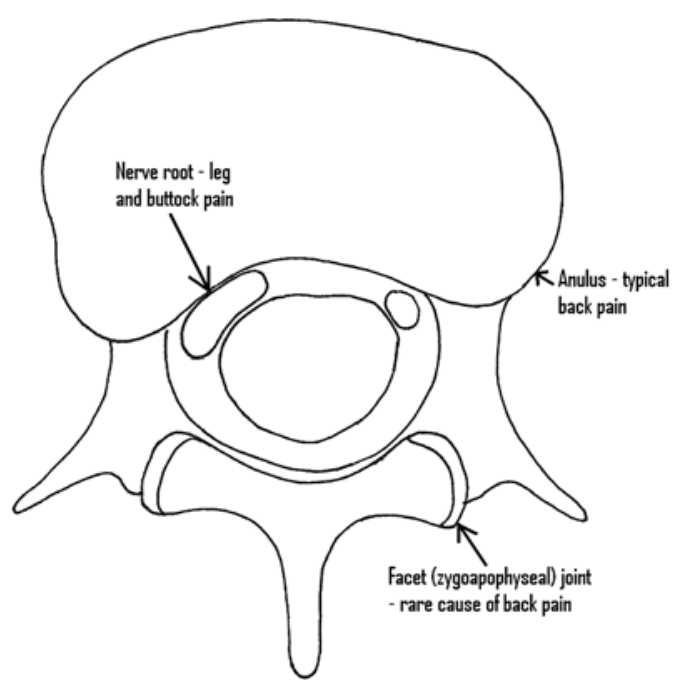

